\title{
Distinct kinesin motors drive two types of maize neocentromeres
}

\author{
Kyle W. Swentowsky, ${ }^{1}$ Jonathan I. Gent, ${ }^{1}$ Elizabeth G. Lowry, ${ }^{1}$ Veit Schubert, ${ }^{2}$ Xia Ran, ${ }^{3,4}$ \\ Kuo-Fu Tseng, ${ }^{3,4}$ Alex E. Harkess, ${ }^{1}$ Weihong Qiu, ${ }^{3,4}$ and R. Kelly Dawe ${ }^{1,5}$ \\ ${ }^{1}$ Department of Plant Biology, University of Georgia, Athens Georgia 30602, USA; ${ }^{2}$ Department of Breeding Research, Leibniz \\ Institute of Plant Genetics and Crop Plant Research, Gatersleben, 06466 Seeland, Germany; ${ }^{3}$ Department of Physics, Oregon State \\ University, Corvallis, Oregon 97331, USA; ${ }^{4}$ Department of Biochemistry and Biophysics, Oregon State University, Corvallis, \\ Oregon 97331, USA; ${ }^{5}$ Department of Genetics, University of Georgia, Athens Georgia 30602, USA
}

A maize chromosome variant called abnormal chromosome 10 (Ab10) converts knobs on chromosome arms into neocentromeres, causing their preferential segregation to egg cells in a process known as meiotic drive. We previously demonstrated that the gene Kinesin driver (Kindr) on Ab10 encodes a kinesin-14 required to mobilize neocentromeres made up of the major tandem repeat knob180. Here we describe a second kinesin-14 gene, TR-1 kinesin (Trkin), that is required to mobilize neocentromeres made up of the minor tandem repeat TR-1. Trkin lies in a 4-Mb region of Ab10 that is not syntenic with any other region of the maize genome and shows extraordinary sequence divergence from Kindr and other kinesins in plants. Despite its unusual structure, Trkin encodes a functional minus end-directed kinesin that specifically colocalizes with TR-1 in meiosis, forming long drawn out neocentromeres. TRKIN contains a nuclear localization signal and localizes to knobs earlier in prophase than KINDR. The fact that TR-1 repeats often co-occur with knob180 repeats suggests that the current role of the TRKIN/TR-1 system is to facilitate the meiotic drive of the KINDR/knob180 system.

[Keywords: meiotic drive; anaphase; chromosome segregation; heterochromatin; kinesin-14; knob; meiosis; neocentromere; tandem repeat]

Supplemental material is available for this article.

Received May 23, 2020; revised version accepted July 28, 2020.

Meiotic drive describes any process whereby a selfish gene, complex of genes, or chromosomal feature reproducibly segregates to progeny at frequencies higher than Mendelian expectations (Fishman and McIntosh 2019). Most are male meiotic drive systems that use genetic elements to alter sperm or pollen viability and do not alter the mechanics of meiosis. In contrast are several female meiotic drive systems that directly impact the segregation of chromosomes (Fishman and Saunders 2008; Chmátal et al. 2014, 2015; Akera et al. 2017; Iwata-Otsubo et al. 2017; Dawe et al. 2018; Wu et al. 2018). Asymmetric segregation in mice is explained by differences in centromeric tandem repeat abundance that causes a preferential orientation of one chromosome toward the egg pole in meiosis I (IwataOtsubo et al. 2017; Wu et al. 2018). In maize, meiotic drive is caused by a chromosome variant, Abnormal chromosome 10 (Ab10), that converts tandem repeat arrays called knobs into spindle attachments called neocentromeres (Rhoades 1942; Longley 1945). According to Rhoades (1952) (Fig. 1A), recombination between centromeres and knobs creates the opportunity for neocentromeres to

Corresponding author: kdawe@uga.edu

Article published online ahead of print. Article and publication date are online at http://www.genesdev.org/cgi/doi/10.1101/gad.340679.120. move preferentially to the basal daughter cell that will ultimately become the female gametophyte that gives rise to the egg cell. Knobs can be tens of megabases in length, are strikingly polymorphic between lines, and exist in an extreme form of tightly condensed heterochromatin $(\mathrm{Mc}-$ Clintock 1929; Albert et al. 2010; Dawe et al. 2018; Liu et al. 2020). The ability of knobs to directly engage meiotic spindles as neocentromeres and undergo meiotic drive explains their prevalence in all maize lines (Buckler et al. 1999).

Recent results revealed that neocentromeres are activated by a kinesin-14 motor on Ab10 called Kinesin-driver (Kindr) (Dawe et al. 2018). Kinesins are a superfamily of microtubule-based motor proteins that display a wide range of functions including vesicle and organelle movement and small molecule transport (Marx et al. 2009; Endow et al. 2010). The kinesin-14s are the only class that moves cargoes toward the minus ends of microtubules that accumulate at spindle poles (Lawrence et al. 2004;

(C) 2020 Swentowsky et al. This article is distributed exclusively by Cold Spring Harbor Laboratory Press for the first six months after the full-issue publication date (see http://genesdev.cshlp.org/site/misc/terms.xhtml). After six months, it is available under a Creative Commons License (Attribution-NonCommercial 4.0 International), as described at http://creativecommons.org/licenses/by-nc/4.0/. 
A

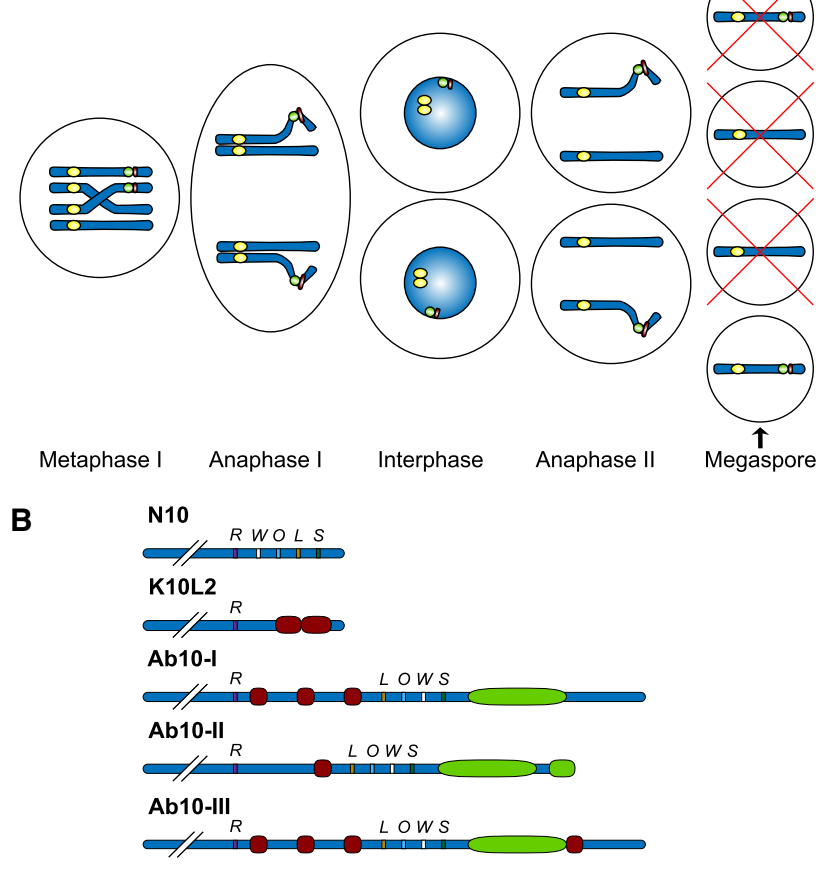

Figure 1. Ab10 types and the Rhoades model for meiotic drive (Rhoades 1952). (A) Schematic of known maize chromosome 10 haplotypes where green and red indicate knob180 and TR-1 knobs, respectively. $\mathrm{R}, \mathrm{W}, \mathrm{O}, \mathrm{L}$, and $\mathrm{S}$ denote relative positions of genetic markers, signifying known inversions that exist between N10 and Ab10. (B) The Rhoades model for neocentromere-mediated meiotic drive (Rhoades 1952). Centromeres are shown in yellow, knob180 are in green, and TR-1 are in red. Recombination must occur between centromeres and knobs to create heteromorphic dyads, where one chromatid contains a knob and the other does not. The extreme poleward orientation of knobs is maintained through meiotic interphase, and in meiosis II, knobs again move toward the upper and lower cells of the linear tetrad. Since the basal cell becomes the megaspore (progenitor of the egg), knobs are preferentially transmitted.

Gicking et al. 2018). The closest homologs of Kindr are maize Variable kernel size1 (Vks1) (Huang et al. 2019) and Divergent spindle1 (Dv1) (Clark 1940; Higgins et al. 2016), both of which function in spindle organization. KINDR differs by binding specifically to knobs and powering them toward spindle poles at both meiosis I and II to mediate their preferential segregation (Dawe et al. 2018).

Knobs can be found at 34 distinct positions and are typically located in midway positions along the chromosome arms in gene-dense areas (Kato 1976; Buckler et al. 1999; Albert et al. 2010; Ghaffari et al. 2013). They are polymorphic in their presence/absence, size, and sequence composition, but most maize lines contain between three and 12 visible knobs (Kato 1976; Albert et al. 2010). At the molecular level knobs are composed of two types of tandem repeats: the more abundant 180-bp tandem repeat (knob180) (Peacock et al. 1981), and the less abundant 359-bp tandem repeat (TR-1) (Ananiev et al. 1998). The KINDR protein localizes specifically to knob180 repeats (Dawe et al.
2018). A subset of the known Ab10 types, including the reference form Ab10-I-MMR, also promote neocentromere activity at TR-1 repeat arrays (Figs. 1B, 2A). TR-1 neocentromeres remain active in kindr mutants, indicating that there are two separate genes that independently control neocentromere activity of knob180 and TR-1 neocentromeres (Hiatt et al. 2002). A factor that controls TR-1 neocentromere activity was previously mapped to a region of Ab10 that contains three TR-1 knobs (Fig. 1B; Hiatt et al. 2002).

Despite the prevalence of TR-1 repeats in Zea and its sister genus Tripsacum, their role in meiotic drive has remained an open question. One hypothesis, that TR-1 knobs are required for meiotic drive, is supported by data showing that a TR-1 knob on the chromosome variant K10L2 displays weak $(51 \%-52 \%)$ meiotic drive and that a TR-1-rich knob on chromosome 6 is preferentially segregated when crossed in the presence of Ab10 (Kanizay

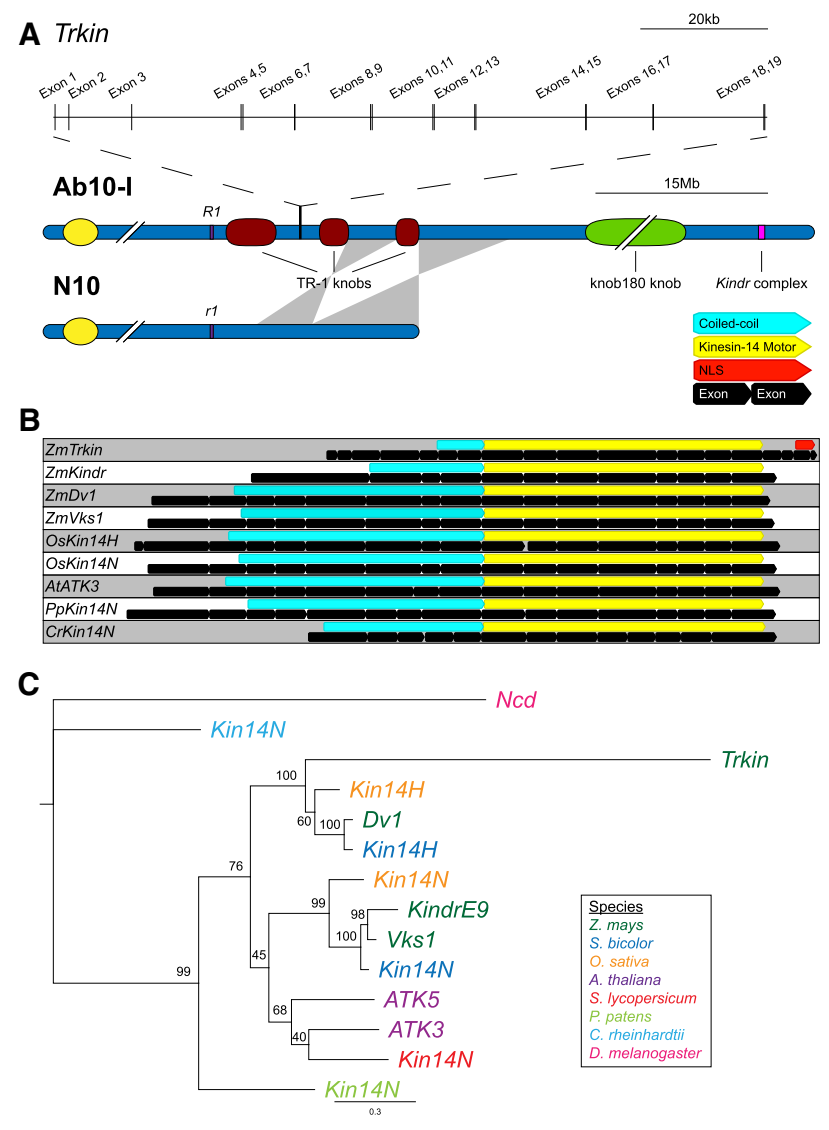

Figure 2. Trkin and its divergence from other kinesin-14s. (A) Comparison of Ab10-I-MMR and normal chromosome 10 from B73 (v5 assembly). Regions of shared, inverted synteny between N10 and Ab10 are highlighted in gray. The $\sim 130-\mathrm{kb}$ Trkin gene is expanded above Ab10. The Kindr complex, highlighted in purple, contains nine Kindr genes in tandem. (B) Alignment of the Trkin gene and selected orthologs. The black boxes show exon boundaries. Predicted protein domains are also highlighted, including coiled-coil (cyan), kinesin-14 motor (yellow), and nuclear localization signal (red). (C) Phylogeny of Trkin and related kinesin-14 motor domains. Bootstrap values are shown at nodes. 
et al. 2013a). A second hypothesis is that TR-1 currently has no role in meiotic drive; this view is supported by the fact that TR-1 neocentromeres are not sufficient to cause meiotic drive of $\mathrm{Ab} 10$ when the KINDR/knob180 system is absent (Dawe et al. 2018). A third view is that the TR-1 neocentromere system functions primarily as an antagonist of Ab10-meiotic drive. Support for the latter view comes from the fact that the TR-1-rich knob on $\mathrm{K} 10 \mathrm{~L} 2$ reduces the meiotic drive of $\mathrm{Ab} 10$ when paired in opposition (Kanizay et al. 2013a).

We recently completed a high quality genome assembly of Ab10 including the distal $53 \mathrm{Mb}$ region containing the meiotic drive haplotype (Fig. 2A; Liu et al. 2020). The assembly gives molecular details to the many known features of $\mathrm{Abl0}$ that were described using classical approaches, including its three TR-1-rich knobs (Hiatt et al. 2002), large knob composed primarily of knob180 repeats (Longley 1938; Peacock et al. 1981), two inversions containing genes shared with N10 (Mroczek et al. 2006), and the nine-gene Kindr complex that is required for knob180 neocentromere activity and meiotic drive (Dawe et al. 2018). Here we demonstrate that the new genome assembly includes another divergent kinesin-14 gene that we call TR-1 kinesin (Trkin). TRKIN is a functional kinesin-14 protein that localizes specifically to TR-1 neocentromeres during meiosis. Analysis of neocentromeres in Ab10 types differing in the presence or absence of Trkin support the view that it is necessary for TR-1 neocentromere formation. Surprisingly, Trkin is not a homolog of Kindr and shows extreme sequence divergence suggestive of an ancient origin. We argue that in modern Ab10 types, the TRKIN/TR-1 system functions primarily to ensure the efficiency and fidelity of meiotic drive caused by the KINDR/knob180 system.

\section{Results}

Trkin encodes a divergent kinesin-14 protein

Whereas Kindr is encoded by nine genes distal to the large knob180-rich knob (Dawe et al. 2018; Liu et al. 2020), cytological analysis of Ab10 deletion derivatives indicated that the TR-1 neocentromere-activating factor is encoded near or among the small TR-1 knobs (Hiatt et al. 2002). In line with expectations, the complete Ab10 assembly revealed an expressed kinesin-14 gene between two of the TR-1 knobs in an $\sim 4-\mathrm{Mb}$ region that is not shared with N10 (Fig. 2A). We named the gene TR-1 Kinesin, or Trkin for short. Trkin is a large gene extending $>130 \mathrm{~kb}$ (Fig. 2A). It has 19 exons, 14 of which are organized in an alternating pattern of short introns followed by long introns. The large introns are composed primarily of transposable elements, including $27 \mathrm{~kb}$ of L1 line elements (Supplemental Fig. S1). To confirm the accuracy of the Trkin annotation, we sequenced 23 cDNA clones. Of these, 22 agreed with the original annotation. The single exception was an alternative splicing event that affected the last exon and a small amount of terminal sequence.

The predicted TRKIN protein contains three known motifs: a short 54-amino-acid coiled-coil domain, a kine- sin-14 motor domain, and a nuclear localization sequence (NLS) (Fig. 2B). Coiled-coil domains in kinesins are usually involved in protein dimerization, but the TRKIN coiled-coil region is unusually small. For comparison with other maize kinesin-14s, the coiled-coil region in KINDR is 128 amino acids, in DV1 it is 308 amino acids, and in VKS1 it is 303 amino acids. The 126 amino acids that comprise the N-terminal end of TRKIN are uncharacterized, but would normally be the location of a cargobinding domain (Marx et al. 2009). The NLS lies in a 68-amino-acid C-terminal extension beyond the motor domain. Neither a C-terminal extension nor an NLS are present in other characterized kinesin-14 homologs (Cross and McAinsh 2014). The 1668-nt Trkin coding sequence does not produce significant DNA alignments outside of its conserved motor domain to annotated maize transcripts or complete maize genomes (except for pseudoTrkin, see below).

To explore the relationship of Trkin to other kinesin14 s, we constructed a maximum likelihood phylogeny using cDNA sequences from the conserved motor domains. Kinesin-14s from angiosperms formed a cluster with two main lineages (Fig. 2C). One of these contains the maize kinesin-14s Kindr and Vks1, which were estimated to have diverged from each other $\sim 12$ million yr ago (Dawe et al. 2018). Another lineage contains Trkin and its closest maize homolog Dv1. It is readily apparent that Trkin is not related by recent ancestry to Kindr. A simple interpretation based on its position within the tree is that the Trkin motor diverged before a split between rice (Oryza sativa) and the panicoid grasses (represented here by maize and S. bicolor) (Fig 2C). Trkin either evolved early in the grass lineage or has rapidly evolved in a short time to become a kinesin-14 that differs significantly from any other kinesins in the grass clade.

\section{TRKIN is a functional kinesin}

The unusually short coiled-coil domain and low amino acid identity between TRKIN and other kinesins raises the question of whether it is a functional kinesin. To determine whether key motifs within the kinesin motor domain are conserved, we compared protein alignments of TRKIN with KINDR, DV1, VKS1, two homologs from rice (OsKIN14H and OsKIN14N), a homolog from Arabidopsis (AtATK3), one from the moss Physcomitrella patens (PpKIN14N), and another from the green algae Chlamydomonas reinhardtii (CHLREDRAFT120667). Despite the fact that the motor domain shows only $43.6 \%$ amino acid identity to its closest maize homolog DV1 (Higgins et al. 2016), the exon positions are conserved (Fig. 2B) and all residues of the ATP binding site are present (Supplemental Fig. S2; Gulick et al. 1998). There is weak similarity to the neck region at the $\mathrm{N}$-terminal end of kinesin-14 motor domains that influence minus end-directed motility (Supplemental Fig. S2; Yamagishi et al. 2016). TRKIN also lacks 16 residues (relative to other plant kinesin-14s) that would comprise the loop-8 motif (Fig. 3A). The loop- 8 motif resides near the microtubule-binding interface (Kozielski et al. 1997; Woehlke et al. 1997) and 
A

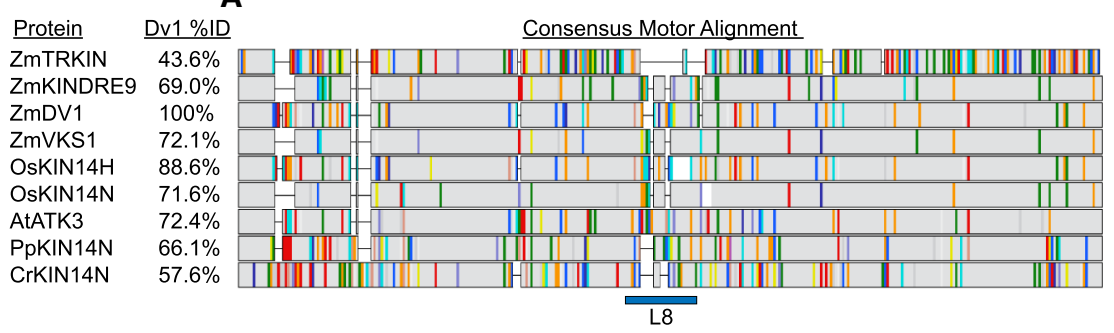

B

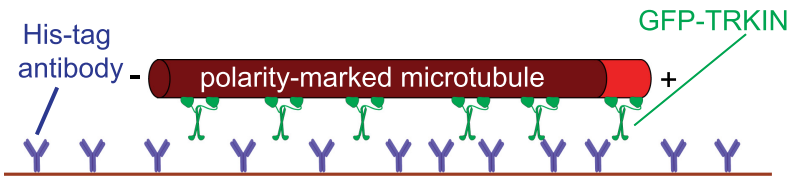

C
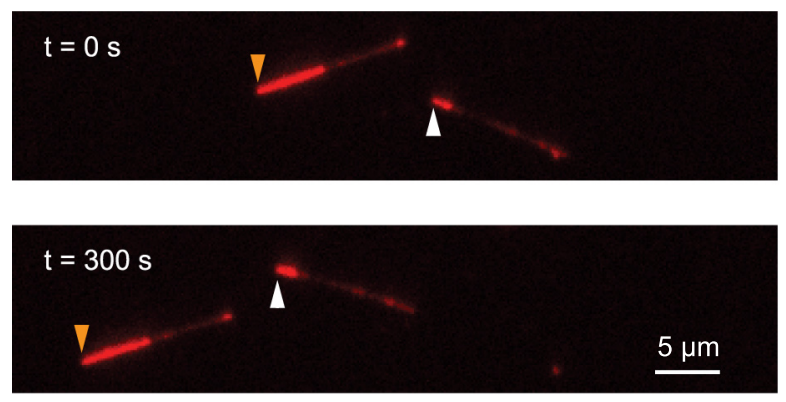

Figure 3. Conservation and functionality of the TRKIN motor. (A) Alignment of the TRKIN motor domain and selected orthologs. Percent identity of each protein to $\mathrm{ZmDV} 1$ is indicated at the left. Sequence matches (in gray boxes), deletions (in black lines), and substitutions (in RasMol colors) are shown relative to the consensus of all proteins in the alignment. The position of the loop-8 motif is highlighted in blue. $(B)$ Schematic of the microtubule-gliding assay. His tag antibodies (blue) adhere to the microscope slide surface and immobilize GFPTRKIN proteins. Motor activity moves the polarity-marked microtubules along the slide. (C) Representative images showing that surface-immobilized GFP-TRKIN causes polarity-marked microtubules to move with the bright plus ends leading; yellow and white arrowheads indicate the plus ends of two different microtubules. mutations affecting this region in the yeast kinesin-5, Cin8, have been shown to alter its microtubule-binding activity (Gerson-Gurwitz et al. 2011; Bell et al. 2017).

To test whether the dramatic sequence divergence of TRKIN affects its ability to function as a minus end-directed motor, we purified a 6xHis/GFP-tagged version of TRKIN and performed in vitro microtubule-gliding experiments using total internal reflection fluorescence microscopy (Fig. 3B). The results revealed that, like KINDR, TRKIN is a functional minus end-directed motor, moving microtubules along a microscope slide with brightly labeled plus ends leading (Fig. 3C). However, TRKIN generates considerably slower $(38 \mathrm{~nm} / \mathrm{sec}$ ) (Supplemental Fig. S3) rates of gliding than KINDR (154 nm/ sec), suggesting that the mechanism of TRKIN movement is different from KINDR. In vivo, TRKIN appears to be at least as effective as KINDR, as TR-1 neocentromeres are long and streaking, often stretching across entire half spindles (Fig. 4A; Hiatt et al. 2002).

\section{TRKIN colocalizes specifically with TR-1 neocentromeres}

Quantitative RT-PCR of cDNA from anther and ear tissue containing meiotic cells revealed Trkin expression (Supplemental Fig. S4). We also observed expression in leaf tissue, similar to what was observed for Kindr (Dawe et al. 2018). To determine the localization of TRKIN in meiosis, we generated antibodies against a peptide corresponding to 19 residues near the N-terminal end of TRKIN (Fig. 4C). We combined TRKIN immunofluorescence with knob fluorescence in situ hybridization (FISH) on male meio- cytes carrying Ab10-I-MMR. Meiotic drive does not occur in male meiosis because all four products of meiosis survive; however, neocentromeres are evident in microsporocytes and are traditionally scored in these cells. The data revealed a clear and precise overlap between TRKIN staining and TR-1 neocentromeres and an absence of staining over knob180 neocentromeres (Fig. 4A; Supplemental Movie S1). As a control we also carried out immunofluorescence on another Ab10 type, Ab10-II-MMR, which lacks TR-1 neocentromeres and appears to lack the N-terminal region that the antibody was generated against (Fig. 4C). As predicted, there was no above-background TRKIN signal in Ab10-II-MMR meiocytes (Fig. 4B).

\section{Chromosome 10 variants that express full-length Trkin show TR-1 neocentromeres}

Ab10 chromosomes have been categorized into groups based on their cytological characteristics: Ab10-I, with three small TR-1-rich knobs and a large knob180-rich knob; Ab10-II, with one TR-1-rich knob and two knob180-rich knobs; Ab10-III, which is similar to Ab10-I but with a large mixed knob (with both types of repeats); and K10L2, a chromosome 10 variant that has a single large TR-1-rich knob (Fig. 1B). Each of the Ab10 types differ in their capacity to activate TR-1 neocentromeres (Kanizay et al. 2013b), suggesting that they also differ in Trkin expression.

Ab10-I-MMR and Ab10-II-MMR are reference forms from Rhoades $(1942,1952)$ that trace to Mexico. Additional variants are from Mexico (Ab10-I-Jal, Ab10-II-Sal, Ab10II-Tel, and Ab10-III-Oax), from Guatemala (Ab10-III-Gua), 
A

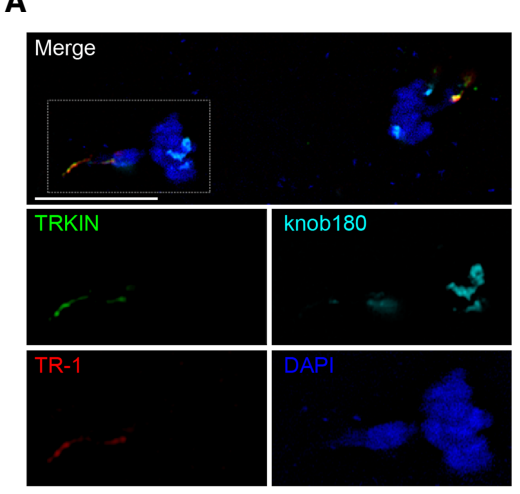

B

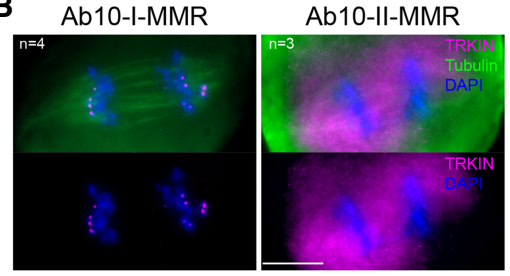

C

C

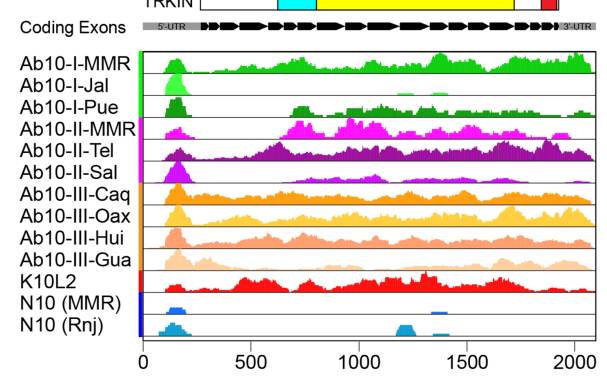

D

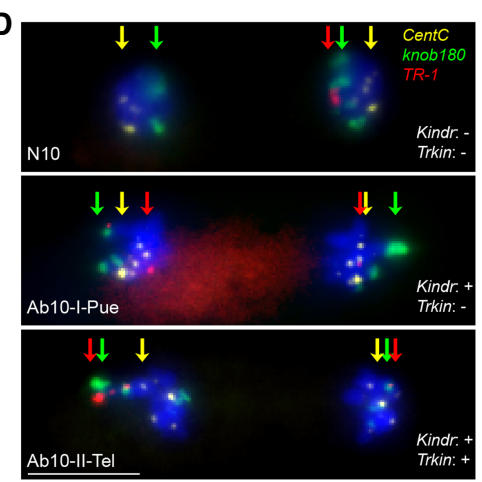

Figure 4. TRKIN localization and presence/absence in different Ab10 types. (A) Visualization of TRKIN during anaphase II by structured illumination microscopy. TRKIN protein immunolocalization (green) and TR1 (red) and knob180 (cyan) sequences labeled by FISH in a line homozygous for Trkin but lacking Kindr. The image is representative of results obtained from eight different plants. Three-dimensional visualization of this cell is in Supplemental Movie S1. (B) Localization of TRKIN (magenta) and tubulin (green) in Ab10-I-MMR and Ab10-II-MMR male meiocytes during anaphase II. Note the absence of specific staining in the Ab10-II-MMR line. The images shown are representative of results obtained from four (Ab10-I) and three (Ab10-II) plants. (C) Distribution of mRNA-seq reads from various libraries aligned to the Trkin transcript. The predicted TRKIN protein and coding exons are shown, along with the location of the peptide used to prepare antibodies (blue) for reference. Track colors reference cytologically distinguishable haplotypes: Ab10-I (green), Ab10-II (purple), Ab10-III (orange), K10L2 (red), and N10 (blue). Vertical black lines indicate mismatched bases. The $Y$ axes are scaled relative to the read count in the various samples and should not be interpreted as absolute values. $(D)$ Correlation between Trkin and TR-1 neocentromeres in different genotypes. Merged FISH images show CentC (yellow), knob180 (green), and TR-1 (red) during anaphase II. Knob positions are denoted by colored arrows. TR-1 neocentromeres generally move faster than knob 180 neocentromeres, and neocentromeres of both types arrive at the poles before centromeres. The images are representative of results obtained from analyzing three plants for each genotype. Scale bars, $10 \mu \mathrm{m}$.

and from Colombia (Ab10-III-Hui and Ab10-III-Caq) (Kanizay et al. 2013b; Higgins et al. 2018). We examined RNAseq data from meiotic anthers taken from each of these haplotypes and wild-type siblings (Higgins et al. 2018), focusing on the genes expressed from the $\sim 53-\mathrm{Mb} \mathrm{Ab} 10$ haplotype (Liu et al. 2020). Hierarchical clustering of the gene expression profiles (Supplemental Fig. S5) revealed that the K10L2 and N10 samples form a distinct cluster, consistent with the previous conclusion that K10L2 is an N10-like chromosome (Kanizay et al. 2013a). All of the Ab10 samples clustered into a single group, with the exception of one Ab10-III-Gua replicate that is probably from an N10 sibling that was mis-genotyped. While the Ab10-I and Ab10-III samples generally clustered together, Ab10-I-Jal clustered with the Ab10-II samples and is likely a chimeric form generated by recombination between Ab10 haplotypes (Kanizay et al. 2013a).

Among the diverse Ab10 types we observed three categories of Trkin expression (Fig. 4C). One group includes Ab10-I-MMR, Ab10-II-Tel, K10L2, and all Ab10-III accessions, which encode a full-length Trkin transcript. The second includes Ab10-I-Pue, Ab10-II-MMR, and Ab10-IISal, which have an apparent truncation of the first five coding exons. The third is Ab10-I-Jal, which lacks Trkin expression altogether. The Trkin expression data correlate with the presence or absence of TR-1 neocentromeres as reported in the prior literature. Both Ab10-I-MMR and K10L2 express a full length Trkin transcript and show
TR-1 neocentromeres (Hiatt et al. 2002; Kanizay et al. 2013a) while Ab10-II-MMR does not express a full length Trkin transcript and does not show TR-1 neocentromeres (Mroczek et al. 2006).

We also scored neocentromeres in Ab10-II-Tel and Ab10-I-Pue by analyzing meiotic anaphase samples. The data reveal that while Ab10-II-Tel is structurally and phylogenetically different from Ab10-I-MMR and K10L2, all three express full length Trkin transcripts and display TR-1 neocentromeres (Fig. 4D). Ab10-I-Pue is structurally and phylogenetically different from Ab10-II-MMR, yet neither express full length Trkin transcripts or show active TR-1 neocentromeres (Fig. 4D). The correlation between Trkin expression and TR-1 neocentromere activity in five diverse lines suggests that Trkin is required for TR-1 neocentromere activity, although we cannot rule out other theoretical possibilities without a Trkin knockout mutation in an isogenic background.

Read alignment revealed that Ab10-I-MMR has two pseudogenes homologous to Trkin. One, pseudo-Trkin1, is structurally similar to Trkin but has numerous SNPs and indels, including an early frameshift in the 2nd exon that would render the transcript noncoding. It is part of an $~ 1-\mathrm{Mb}$ inverted duplication encompassing Trkin (Supplemental Fig. S6). The other, pseudo-Trkin2, is located in a region of $\mathrm{Ab} 10$ that is shared with normal chromosome 10 (Figs. 1B, 2A), though nonrecombining due to two large inversions. The maize inbred B73 carries a similar pseudo- 
Trkin in a position syntenic with Ab10 pseudo-Trkin2 (Supplemental Fig. S6). Transcription of pseudo-Trkin could explain the apparent heterozygosity and nonuniform coverage over Trkin mRNA in some accessions (Fig. 4C).

\section{TRKIN localizes to knobs earlier in meiosis than KINDR}

The availability of specific antibodies to TRKIN and KINDR made it possible to compare their localization patterns during each stage of male meiosis in Ab10-I-MMR plants. TRKIN localized to a few small puncta on chromosomes throughout meiosis I, meiotic interphase, and meiosis II (Fig. 5). It was first visible before nuclear envelope breakdown in the pachytene substage of prophase I, suggesting that the predicted NLS in TRKIN is functional. In contrast, KINDR staining was not visible on knobs until after nuclear envelope breakdown. Both TRKIN and KINDR stained brightly throughout metaphase I, anaphase I, and telophase I (Fig. 5), and were detectable in meiotic interphase where they localized inside the nucleus at the site of the latent spindle poles. Both proteins were undetectable after the completion of telophase II. These data are consistent with the hypothesis that both TRKIN and KINDR serve as molecular motors for neocentromere motility, and suggest that TRKIN may be active in the earliest stages of spindle morphogenesis.

\section{TR-1 sequences are conserved in Zea and Tripsacum and often occur in mixed arrays}

One way to interpret the function of Trkin is to measure its impact on the sequence and distribution of TR-1 repeats throughout the genome. Long repeat arrays are inherently unstable because of illegitimate recombination (GarridoRamos 2017), and in the absence of selection, should accumulate sequence variants and ultimately be lost (Charlesworth et al. 1994). Both TR-1 and knob180 are present throughout Zea and within its close relative Tripsacum dactyloides (Albert et al. 2010). To explore the variation among TR-1 sequences we produced TR-1 consensus sequences for individual plants from Zea mays, Zea luxurians, and Tripsacum dactyloides using short reads from previously published studies (Novák et al. 2010; Gent et al. 2017). The TR-1 consensus sequences were similar among species with the consensus in Z. mays being $95 \%$ identical to T. dactyloides (Fig. 6A). For comparison, the knob180 consensus sequence in $Z$. mays has $99 \%$ identity with $T$. dactyloides and the centromere repeat CentC consensus has $97 \%$ identity with $T$. dactyloides (Gent et al. 2017). Mapping short reads to the consensus sequences revealed that the majority of TR-1 sequences were more than $90 \%$ identical to the consensus in each case (Fig. 6B), consistent with a repeat involved in meiotic drive with selection on the primary sequence. Although TR-1 is abundant in T. dactyloides, we were unable to find Trkin in publically available Illumina reads from this species, although it may be present in other accessions that have not yet been sequenced (data not shown).
Ab10 and many other knobs appear to contain both TR-1 and knob180 at the level of FISH (Albert et al. 2010; Kanizay et al. 2013a). To get a more detailed picture of knob mixing, we examined knobs and knob fragments that were assembled in the recently completed genomes of B73 and 25 other inbred lines known as the NAM founders (https://nam-genomes.org/). The data revealed that most knobs contain a significant amount of both repeat types. Only considering arrays of at least $100 \mathrm{~kb}$ in length, mixed knobs were the most common type with a median of nine mixed arrays per line, compared with three containing knob180 alone and two with TR-1 alone (Fig. $6 \mathrm{C})$. The number of mixed arrays is likely higher, because most large knob180 knobs were not completely assembled and may contain TR-1 repeats that were not detected. These data support the prior assertion that the TRKIN/ TR-1 system may be most effective in mixed knobs (Kanizay et al. 2013a).

\section{Discussion}

The plant kinesin-14 superfamily normally functions in spindle and phragmoplast morphogenesis, with lesser roles in nuclear migration and plastid distribution (Gicking et al. 2018). In keeping with these known roles, two native maize kinesin-14s function to focus meiotic spindle poles (Dv1) and ensure accurate spindle formation in endosperm (Vks1) (Higgins et al. 2016; Huang et al. 2019). Kindr shares a recent ancestor with $V k s 1$, yet its function is to bind knob180 repeats and facilitate their segregation as neocentromeres to egg cells (Dawe et al. 2018). Here we describe Trkin as a fourth member of this clade and show that its function is to mobilize TR-1 repeats. The likely existence of Trkin among the three TR-1-rich knobs on Ab10 was predicted by early mapping studies of the TR-1 neocentromere phenotype (Hiatt et al. 2002). That Trkin might be a kinesin was a natural expectation after discovering that Kindr is a kinesin-14 motor (Dawe et al. 2018). However, we were surprised to discover that Trkin is not related to Kindr by descent. The encoded protein shows a highly unusual structure that distinguishes it from all other known kinesin-14 proteins (Figs. 2B, 3A).

Like KINDR, TRKIN is a functional kinesin-14 motor that interacts with a unique class of knob repeats at meiosis. The binding of kinesin to chromatin is remarkably sequence-specific for both the KINDR/knob180 and TRKIN/TR-1 systems, yet we do not understand how this selectivity is achieved. KINDR and TRKIN may either bind directly to DNA or bind as a complex with other proteins that are sequence-specific. The interaction occurs primarily at metaphase and anaphase when neocentromeres are visibly active (Fig. 5). However, unlike KINDR, TRKIN has a nuclear localization signal and can be seen associating with knobs in late prophase I (Fig. 5). TR-1 neocentromeres are visible immediately upon nuclear envelope breakdown, whereas knob180 neocentromeres are slower to move (Hiatt et al. 2002). Another notable difference is that TR-1 neocentromeres appear to uncoil as they move from the metaphase plate to the 


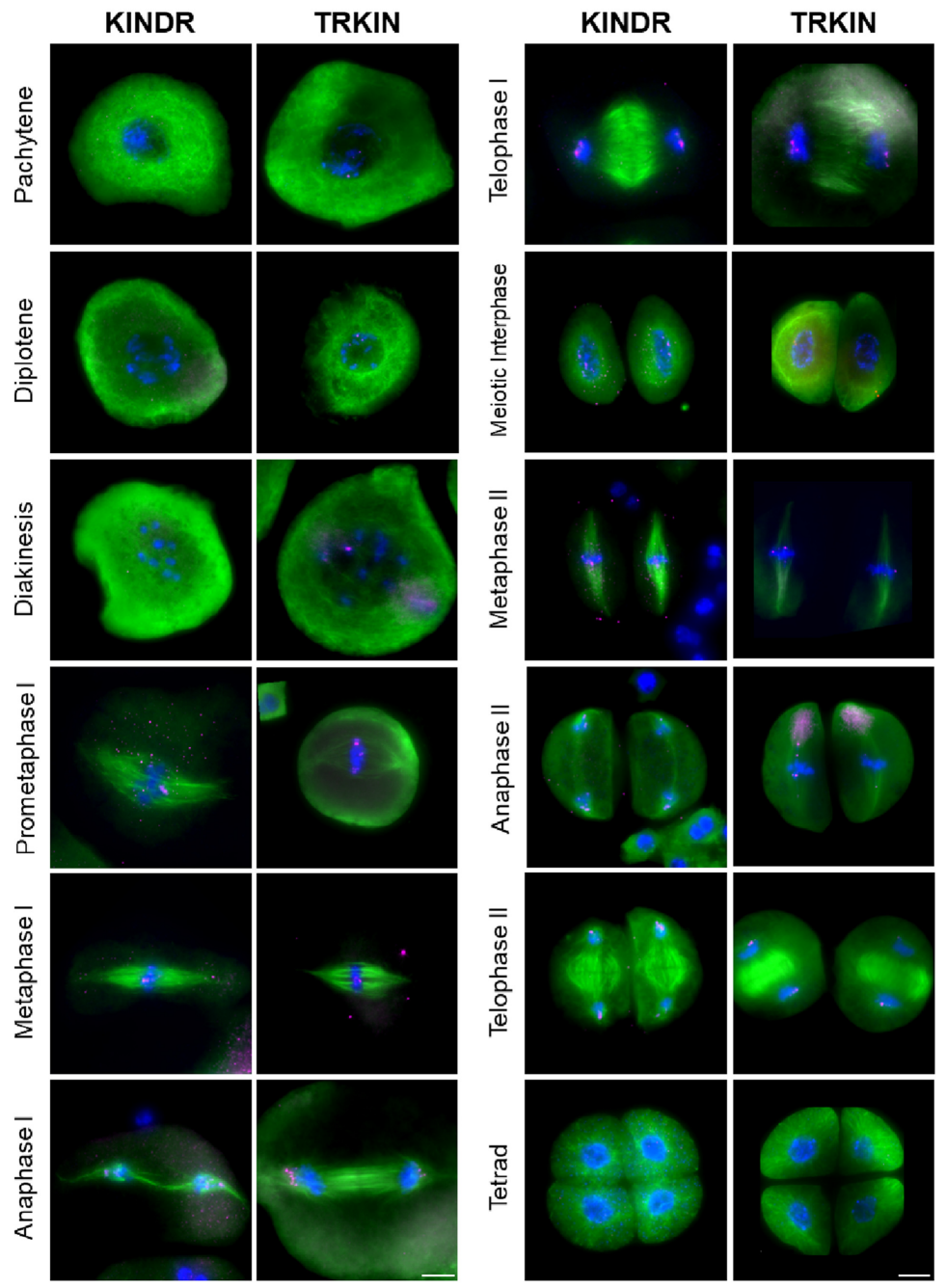

Figure 5. Immunolocalization of TRKIN and KINDR in male meiosis. TRKIN and KINDR are shown in magenta and tubulin is shown in green. The stages of meiosis are indicated at left. The images shown are representative of data collected from eight plants. The TRKIN staining at spindle poles in prometaphase I is background staining and was not observed in all images. Scale bars, $10 \mu \mathrm{m}$.

spindle pole, while knob180 neocentromeres retain their compact structure (Hiatt et al. 2002). The difference in appearance may reflect the unique properties of TRKIN or the genomic constitution of TR-1 arrays, which have far more retrotransposon insertions than knob180 arrays (Liu et al. 2020).

It remains unclear whether the TRKIN/TR-1 neocentromere system can confer meiotic drive on its own. When Kindr is absent, the TRKIN/TR-1 system is not sufficient to cause meiotic drive. However, the K10L2 variant, which does not have Kindr or knob180 repeats, shows a low level of meiotic drive $(\sim 51 \%-52 \%)$. The transmission of K10L2 was statistically different from $50 \%$ in three independent experiments where $>10,000$ kernels were counted (Kanizay et al. 2013a). The fact that K10L2 can compete with Ab10 when paired in opposition also supports the view that the TRKIN/TR-1 system is capable of supporting a low level of meiotic drive (Kanizay et al. 2013a). It is possible that the extreme elasticity of TR-1 neocentromeres reduces their efficiency as neocentromeres. If the elasticity is caused by transposon insertions that reduced the density of TR-1 repeats (Liu et al. 2020), it is reasonable to suggest that the TRKIN/
TR-1 system was once a more powerful meiotic drive system but is now losing its effectiveness.

The current prevalence of mixed knobs suggests there is a benefit to having both work together. A likely scenario is that the early prophase movement and/or inherent elasticity of TR-1 neocentromeres enhances the accuracy of the primary KINDR/knob180 system, while the KINDR/knob180 system assures a high level of meiotic drive for the TR-1 system. One way that the TRKIN/TR1 system may facilitate meiotic drive is by helping to resolve directional conflicts. Classic literature on dicentric chromosomes (McClintock 1939, 1941; Novitski 1952) suggests that linked centromeres on dicentric chromosomes are as likely to move in opposite directions as they are to move in the same direction on the bipolar spindle. Linked knobs and centromeres should behave similarly. This problem is particularly acute in meiosis I where neocentromeres are physically separated from centromeres by chiasmata, which are held in place by sister chromatid cohesion (Fig. 7). Sister chromatids do not separate until anaphase, at which point the centromeres are already in motion (Dawe 1998; Buonomo et al. 2000). Both chromatids may move to the same pole while their 
A A
TR-1 (T. dactyoides)
TR-1 (Z. luxurians)
TR-1 (Z. mays B73)

B

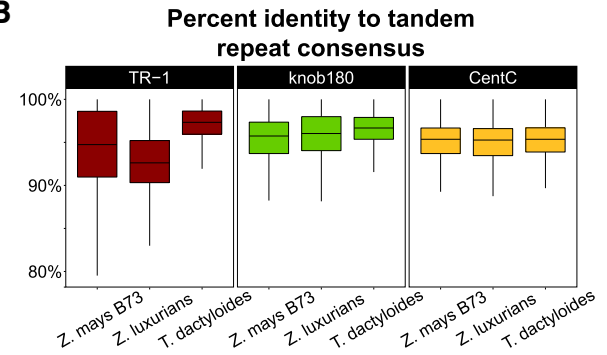

C

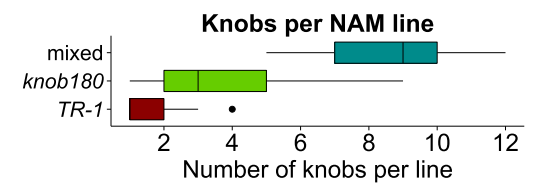

Figure 6. TR-1 sequence diversity and distribution. (A) Multiple sequence alignment of TR-1 consensus sequences made from T. dactyloides, Z. luxurians, and Z. mays (B73). Vertical colored bars indicate SNPs and horizontal lines indicate deletions relative to the consensus made by the three sequences. $(B)$ Sequence variation within repeat families. Illumina reads were mapped to the consensus sequences and the distribution of percent identities plotted as boxplots. (C) The number of mixed (knob180 and TR-1), knob180-only, and TR-1-only repeat arrays in B73 and 25 other sequenced maize genomes plotted as box plots. For box plots in $B$ and $C$, the bounds of boxes indicate first and third quartiles and whiskers show the distribution of 1.5 interquartile range.

respective centromeres migrate in opposite directions, potentially causing chromosome lagging and breakage. However, since bridges and breakage are not regularly observed in Ab10 lines (Yu et al. 1997), the neocentromere traveling in the direction opposite to the centromere must switch directions. For the same reasons, directional conflicts are likely in meiosis II when both sister chromatids contain knobs. It is possible that TRKIN has a higher microtubule dissociation rate than KINDR and can change direction more effectively.

Some mouse strains demonstrate a process known as centromere drive where larger centromeres preferentially engage with the egg pole side of the spindle, which differs by having more tyrosinated tubulin (Akera et al. 2017, 2019). This raises the question of whether there is any asymmetry during maize female meiosis. Indeed, there is visible asymmetry after both meiosis I and meiosis II, where the basal cells are larger (Evans and Grossniklaus 2009). There are also differences in callose deposition (a cell wall component) and the number of plasmodesmata (connections between cells) (Russell 1979). An asymmetrically distributed molecule, most likely one related to the spindle, could in theory help direct KINDR and TRKIN toward the basal cell in meiosis I or II. However, as originally postulated, the Rhoades model for meiotic drive in maize (Rhoades 1952) requires neither spindle asymmetry nor other molecular asymmetry. According to this view, meiosis I simply sets the stage by pulling all knobs toward the polar periphery of telophase nuclei (Fig. 1A), where they appear (at least in male meiosis) to stay throughout interphase (Dawe and Cande 1996). The peripheral location has been postulated to facilitate the movement of knobbed chromatids to the basal cell in meiosis II (Yu et al. 1997; Dawe and Hiatt 2004) as a natural outcome of Ran-regulated spindle self-organization (Zhang and Dawe 2011). As the spindle forms around chromatin, basally oriented neocentromeres could apply tension to the linked kinetochores such that knobbed chromatids are more likely to develop a connection to the spindle facing the basal cell than their corresponding knobless sister chromatids (Fig. 7). The fact that TRKIN contains a nuclear localization signal whereas KINDR does not suggests that it may be specially adapted to this key early movement event.

In summary, our results support the conclusion that KINDR functions as the primary driver (Dawe et al. 2018) while TRKIN serves to improve the efficiency of drive and/or limit the negative consequences of Ab10 on the organism. Our data showing that the majority of knobs contain both knob180 and TR-1 repeats argues against the proposal that TR-1 is under selection primarily an antagonist of the KINDR/knob180 system, and supports the view that the two repeats cooperate to promote meiotic drive (Kanizay et al. 2013a). Further progress in understanding the role of TRKIN will require making a trkin knockout on an otherwise intact Ab10 chromosome. A trkin knockout may reduce meiotic drive, but based on our results showing that multiple Ab10 chromosomes naturally lack Trkin, we believe the effects will be minimal. The more important role of Trkin may be to reduce the frequency of anaphase errors that cause chromosome loss and reduced fertility. Mathematical modeling suggests that high pollen and ovule viability when Ab10 is heterozygous is critical for the drive system to spread into new populations and maintain a stable equilibrium (Hall and Dawe 2018). Careful analyses of meiotic anaphase in Ab10 lines with and without Trkin, as well measurements of pollen viability and seed set, will be needed to fully test this hypothesis.

\section{Materials and methods \\ Genome references and annotation}

The Trkin gene was annotated as a part of the Ab10 assembly project (Liu et al. 2020). Sequences from genomic regions containing pseudo-Trkin arrays were extracted from the maize Ab10 assembly (Liu et al. 2020) and B73v5 assembly (https://www.maizegdb .org/genome/assembly/Zm-B73-REFERENCE-NAM-5.0). Knob composition data were obtained from publicly available maize NAM genome assemblies (https://jbrowse.maizegdb.org/? data=all). Trkin cDNA sequence is available on NCBI GenBank (accession no. MT459824).

$m R N A$ sequencing and analysis

All mRNA samples were derived from whole anthers at the meiotic stage $(2-3 \mathrm{~mm})$ and Illumina sequenced with paired-end 75 - 

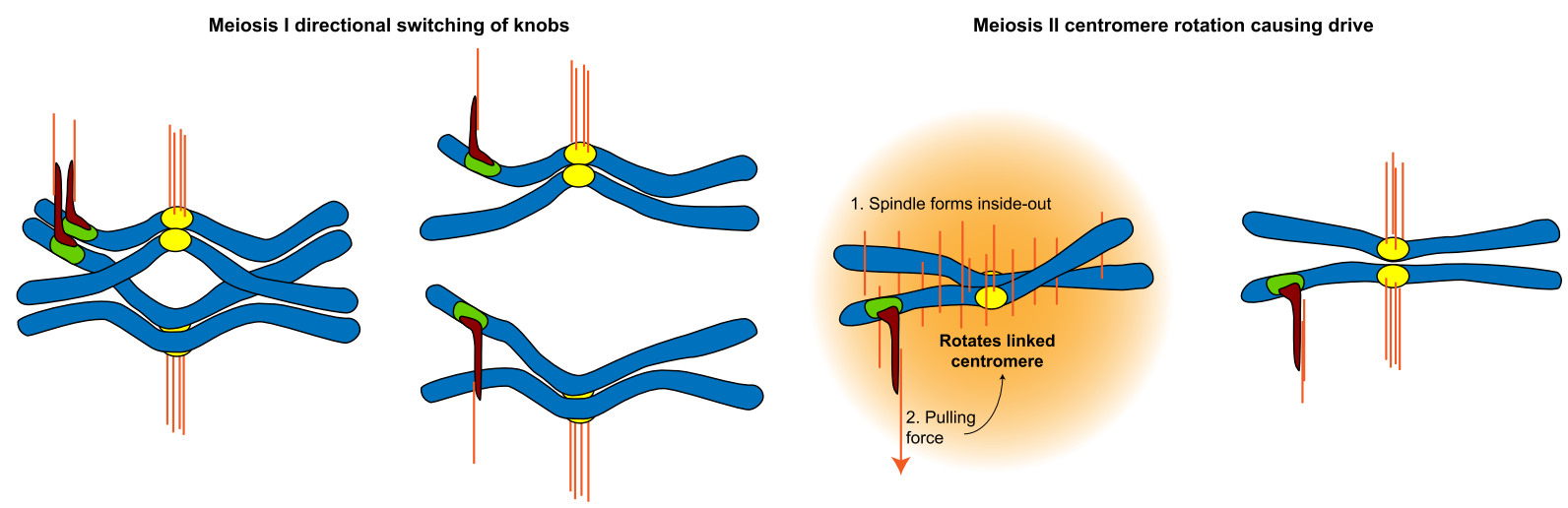

Figure 7. Possible TR-1 neocentromere functions. (A) Meiosis I directional switching. (Left) During meiosis I, sister chromatids remain attached along their entire lengths by cohesin (Dawe 1998; Buonomo et al. 2000). Because they are physically attached, the knobs on sister chromatids are likely to migrate toward one pole, creating a knob-centromere conflict on one pair of homologs. The unusual properties of TRKIN may help resolve these conflicts by facilitating a directional switch. (B) Meiosis II centromere rotation. Neocentromeres in meiosis I leave knobs near the nuclear periphery during interphase, already prepositioned to move toward the basal cell of the meiotic tetrad (see Fig. 1A). The meiotic spindle forms by a self-organization mechanism (Zhang and Dawe 2011). Microtubules are stabilized around chromatin where RanGTP (orange) concentration is highest, and the spindle grows out to form poles. We proposed that neocentromeres move with the forming spindle and rotate linked centromeres in the same direction before stable kinetochore-microtubule contacts are made (Yu et al. 1997; Dawe and Hiatt 2004). The fact that TR-1 neocentromeres move early and fast may facilitate this event.

nt reads, as described previously (Higgins et al. 2018). In addition to the seven accessions from the previous study (NCBI BioProject PRJNA339442), 10 additional chromosome 10 variant genotypes were used for this study (NCBI BioProject PRJNA285341). These included six N10, one K10L2, three Ab10-I, three Ab10-II, and four Ab10-III accessions that are listed along with their SRR identifiers in Supplemental Table S1. For each Ab10 type, we collected data from a minimum of three different plants. For the analysis of Trkin transcript levels among various genotypes, the following protocol was used. Replicates were pooled and mapped to the Trkin cDNA sequence using BWA MEM v0.7.15 (Li 2013) with default parameters. Mapped reads were filtered to allow up to one mismatch in samples known to contain a SNP (Ab10-I-Pue, Ab10-II-MMR, Ab10-II-Tel, Ab10-II-Sal, and K10L2) but no mismatches were allowed for all other samples. Reads were sorted using SAMtools sort v1.3.1 ( $\mathrm{Li}$ et al. 2009) and exported for visualization in IGV v2.6.3 (Robinson et al. 2011).

For expression clustering analysis, reads were initially trimmed using Sickle v1.33 (https://github.com/najoshi/sickle) and mapped to the Ab10 reference using default parameters in the STAR v2.7.1 package (Dobin et al. 2013). Gene expression in TPM was calculated using the package TPMCalculator v0.2 (Vera Alvarez et al. 2019). TPM values were imported into RStudio for analysis, and only transcripts present in the Ab10 haplotype region (after coordinate $135 \mathrm{Mb}$ ) were retained for analysis. The heatmap.2 function in gplots (https://rdrr.io/cran/gplots) was used for hierarchical clustering and heat map visualization.

\section{Analysis of Trkin orthologs}

Trkin homologs from other species were identified by BLAST (NCBI) using the Trkin cDNA sequence as a query. The gene with the best match was chosen for comparison. Gene sequences and their predicted proteins were aligned in Geneious Prime 2019.2.3 (http://www.geneious.com) using the default MUSCLE alignment options.

For phylogenetic gene tree construction, kinesin-14 motor domain-coding sequences were aligned in Geneious Prime 2019.2.3 using the default MUSCLE alignment options. The alignment was uploaded into IQ-TREE (Minh et al. 2020) and a phylogeny was generated using maximum likelihood gene tree construction using the GTR $+\gamma+\mathrm{i}$ models $(\mathrm{GTR}+\mathrm{G}+\mathrm{I}+\mathrm{F} 4$ options) to account for rate heterogeneity.

\section{Analysis of pseudo-Trkin homologs}

Positions of pseudo-Trkin exon orthologs in Ab10 and B73v5 were determined using NCBI Blast v2.2.26. Large genomic sequences were aligned in MiniMap2 (Li 2018) using the -cx asm5 option. Alignments with a quality score of $\geq 60$ were retained for analysis. Genomic alignments were visualized along with pseudo-Trkin exon alignments using the geom_point function in ggplot2 (https://ggplot2.tidyverse.org).

\section{TR-1 consensus sequences}

Consensus sequences for TR-1 were derived from 150-nt singleend Illumina reads from micrococcal nuclease-digested chromatin from a previous study (Gent et al. 2017). SRA run accession numbers are as follows: Zea mays (B73), SRR5466391; Zea luxurians (PI 422162), SRR5466389; and Tripsacum dactyloides (PI 421612), SRR5466393. Consensus sequences were produced with RepeatExplorer (version 2018.11.09) (Novák et al. 2010) using parameters "-max_memory 500000000 -tax VIRIDIPLANTAE3.0 -opt ILLUMINA." Sample-specific consensus sequences were aligned to each other with Geneious Prime 2019.2.3 (http ://www.geneious.com). The percent identity of individual reads with consensus sequences was determined using blastall with parameters as follows: "-p blastn -e 1e-5 -W 7 -G 2 -E 1 -r 1 -q -1." Only reads producing alignments of at least $125 \mathrm{bp}$ in length to consensus sequence dimers were included.

\section{Analysis of knob composition in NAM lines}

Tandem repeat array data for the 26 maize NAM founder genome assemblies were annotated as part of the NAM sequencing project (https://nam-genomes.org//). Here "arrays" are defined as large areas of assemblies composed primarily of short tandem 
repeats but may also be interspersed with other sequences. These data were filtered to only include arrays with $>100 \mathrm{~kb}$ of tandem repeat sequences. Only arrays within the final chromosome assemblies (pseudomolecules) were considered. Knob fragments that were not assigned to a chromosome were not included in the analysis. Knobs were called "knob180" or "TR-1" if $>95 \%$ of the knob repeats in the array were composed of one type of knob repeat. Knobs were called "mixed" if they contained both repeats and one type made up $<95 \%$ of the total.

\section{Plant materials}

The immunolocalization of TRKIN in Figure 4A was performed on a heterozygous K10L2/Ab10-Df(L) line that is homozygous for Trkin but lacks Kindr (Dawe et al. 2018). All other lines used for immunolocalization or FISH (Ab10-I-MMR, Ab10-IIMMR, Ab10-I-Pue, and Ab10-II-Tel) were described previously (Higgins et al. 2018) and heterozygous with N10.

\section{TRKIN antibody preparation}

Antibodies were prepared by Pacific Immunology. The peptide TRKIN(6-24): RGEEPKVVAHREDIKAQFK-Cys was injected into rabbits and antibodies were affinity-purified using the same peptide.

\section{Quantitative RT-PCR}

cDNA samples from Ab10 and N10 (B73 inbred) ears, anthers, leaf tips, and leaf bases originally described in Dawe et al. (2018) were assayed for the presence of Trkin using the SYBR Green PCR master mix (Applied Biosystems 4367659) and assayed in a C1000 Touch thermal cycler with CFX96 real-time system (Bio-Rad 1855195). Trkin primers were designed to capture the splice junction between exons 13-14: Trkin qPCR F1 (5'-GGAGGCCAA GGCCATAAAT- $\left.3^{\prime}\right)$ and Trkin qPCR R1 (5'-TGAGACAGAGTC GATCCTCTAAA- ${ }^{\prime}$ ). Control primers were from the LUG gene (Manoli et al. 2012): LUGF (5'-TCCAGTGCTACAGGGAA GGT-3') and LUGR (5'-GTTAGTTCTTGAGCCCACGC-3'). PCR was performed using an initial denaturing step for $10 \mathrm{~min}$ at $95^{\circ} \mathrm{C}$ followed by 40 cycles of denaturation $\left(15 \mathrm{sec}\right.$ at $\left.95^{\circ} \mathrm{C}\right)$ and annealing/extension $\left(1 \mathrm{~min}\right.$ at $\left.60^{\circ} \mathrm{C}\right)$. Expression was measured from three different plants per tissue and error bars represent biological variation.

Immunolocalization and fluorescence in situ hybridization (FISH)

Immunolocalization was performed as described in Dawe et al. (2018). Briefly, tassels were removed from plants and meiotic anthers were fixed in $4 \%$ paraformaldehyde in PHEMS buffer and $1 \%$ Triton X-100 for $1 \mathrm{~h}$. Meiocytes were extruded from anthers and immobilized onto polylysine-coated coverslips by centrifugation at $100 \mathrm{~g}$ for $1 \mathrm{~min}$. Coverslips were incubated in a permeabilization solution for $1 \mathrm{~h}$ and then in a blocking solution containing $10 \%$ goat serum diluted in PBS for $2 \mathrm{~h}$. Antibodies were diluted in an antibody dilution buffer $3 \%$ BSA diluted in PBS) and incubated overnight at $37^{\circ} \mathrm{C}$. The primary antibodies were $\mathrm{mAb}$ mouse antitubulin (Asai et al. 1982) diluted 1:200, pAb rabbit anti-KINDR (Dawe et al. 2018) diluted 1:100 or $1: 250$, and the pAb rabbit anti-TRKIN described above diluted $1: 100$. An additional blocking step was performed as above then coverslips were incubated in a secondary antibody solution containing rhodamine-conjugated AffiniPure donkey antirabbit IgG $\mathrm{H}+\mathrm{L}$ (Jackson 711-025-152) and fluorescein-conjugated AffiniPure goat antimouse IgG H + L (Jackson 115-095-146), both dilut- ed 1:200 for $2.5 \mathrm{~h}$ at room temperature in the dark. The coverslips were mounted on microscope slides using ProLong Gold with DAPI (Thermo Fisher P36931) before imaging on a Zeiss Axio Imager.M1 fluorescence microscope with a $63 \times$ plan-apo Chromat oil objective, and data were analyzed using Slidebook software (Intelligent Imaging Innovations).

Combined FISH immunolocalization for Figure 4A was performed as described in Dawe et al. (2018). Briefly, coverslips containing fixed meiocytes were suspended over a slide using broken bits of coverslips and a solution containing oligo probes (FITC-labeled 180-bp repeat oligos and rhodamine-labeled TR-1 repeat oligos) (see Kanizay et al. 2013b) was pipetted under the coverslip and the edges were sealed using nail polish. The slides were heated for $5 \mathrm{~min}$ at $95^{\circ} \mathrm{C}$ and then incubated for $2 \mathrm{~h}$ at room temperature in the dark. The coverslips were removed and washed, and immunolocalization was performed as described above using the primary rabbit anti-TRKIN antibody and secondary Cy5 AffiniPure donkey antirabbit IgG H + L (Jackson 711-175-152). Coverslips were mounted on slides using ProLong Gold with DAPI (Thermo Fisher P36931) and imaged using structured illumination microscopy.

FISH for Figure 4D was performed as described in Dawe and Cande (1996). Briefly, coverslips containing fixed meiocytes were incubated with the following solutions for $10 \mathrm{~min}$ each: $1 \times \operatorname{SSC}(4.38 \mathrm{~g} /$ liter sodium citrate $/ 8.75 \mathrm{~g} /$ liter $\mathrm{NaCl}) / 20 \%$ formamide, $2 \times$ SSC $/ 30 \%$ formamide, and $2 \times$ SSC/50\% formamide. Broken bits of coverslips were placed at four corners of a slide, and the coverslips with meiocytes were placed upside down over the broken pieces. Seventy-five microliters of a solution containing $2 \times$ SSC $/ 50 \%$ formamide and $1 \mu \mathrm{g} / \mathrm{mL}$ fluorescently labeled oligos (FITC-labeled 180-bp repeat oligos, rhodaminelabeled TR-1 repeat oligos, Cy5-labeled CentC repeat oligos) (see Kanizay et al. 2013b) was pipetted under the coverslip and the edges were sealed with rubber cement. The slide was placed for $5 \mathrm{~min}$ on a $95^{\circ} \mathrm{C}$ heating block and the probes were allowed to anneal overnight at room temperature. The rubber cement was removed, and the coverslip was incubated with the following solutions for $10 \mathrm{~min}$ each: $2 \times \mathrm{SSC} / 20 \%$ formamide $/ 0.01 \%$ Tween-20, $1 \times$ SSC/10\% formamide/0.01\% Tween- $20,1 \times$ SSC/ $1 \times$ PBS, $1 \times$ PBS, and $1 \times$ PBS $/ 0.1 \mu \mathrm{g} / \mathrm{mL}$ DAPI. The coverslips were mounted on microscope slides and imaged on a Zeiss Axio Imager.M1 fluorescence microscope as described above.

\section{Structured illumination microscopy}

Superresolution microscopy was used to analyze the spatial arrangement of TRKIN, TR-1, and knob180 in Figure 4A. Spatial structured illumination microscopy (3D-SIM) was applied using a plan-apochromat $63 \times / 1.4$ oil objective of an Elyra PS.1 microscope system and the software ZENblack (Carl Zeiss GmbH). Image stacks were captured separately for each fluorochrome using 642-, 561-, 488-, and 405-nm laser lines for excitation and appropriate emission filters. The 3D-SIM stacks with a step size of 110 $\mathrm{nm}$ were acquired consecutively for each fluorophore starting with the highest wavelength dye to minimize bleaching. Maximum intensity projections were calculated based on the image stacks using the ZENblack software (Weisshart et al. 2016). The same image stacks were also used to produce $3 \mathrm{D}$ movies by the Imaris 8.0 software (Bitplane).

TRKIN protein purification and microtubule-gliding experiments

An E. coli codon-optimized version of the Trkin CDS was synthesized by Genscript and cloned into the expression vector pET-17b (Novagen) modified to include $\mathrm{N}$-terminal 6xHis and GFP tags for 
affinity protein purification. Subsequent steps were performed as described in Dawe et al. (2018). Briefly, expression was induced in BL21(DE3) Rosetta cells (Novagen 70954-3) with 0.1 mM IPTG for $12-14 \mathrm{~h}$ at $20^{\circ} \mathrm{C}$. Cells were centrifuged and resuspended in $50 \mathrm{mM}$ sodium phosphate buffer $(\mathrm{pH} 7.2)$ containing $250 \mathrm{mM}$ $\mathrm{NaCl}, 1 \mathrm{mM} \mathrm{MgCl} 2,0.5 \mathrm{mM}$ ATP, $10 \mathrm{mM} \beta$-mercaptoethanol, $20 \mathrm{mM}$ imidazole and a protease inhibitor cocktail, and then lysed by sonication. The lysate was centrifuged and soluble protein in the supernatant was purified by Talon resin (Clontech) and eluted in $50 \mathrm{mM}$ sodium phosphate buffer described above, but with $250 \mathrm{mM}$ imidazole. Protein was flash-frozen and stored at $-80^{\circ} \mathrm{C}$.

In vitro gliding assays were performed as described in Dawe et al. (2018). Briefly, polarity-labeled microtubules were first prepared as described in Hyman (1991). Time-lapse microtubule gliding experiments were performed at room temperature using a Zeiss Axio Observer Z1 objective-type TIRF microscope equipped with a $100 \times 1.46$ NA oil immersion objective and a back-thinned electron multiplier CCD camera (Photometrics). Flow chambers were made by attaching a coverslip to a glass slide by double-sided tape as described in (Popchock et al. 2017) and the chamber was perfused with an anti-His antibody diluted in BRB12 buffer and incubated for $2 \mathrm{~min}$ at room temperature. Unbound antibody was washed away and one chamber volume of 6xHis-TRKIN diluted in BRB50 supplemented with $20 \mu \mathrm{M}$ taxol and $1.3 \mathrm{mg} / \mathrm{mL}$ casein was added to the chamber. Following 2 min of incubation, unbound protein was washed away using BRB50 supplemented with $20 \mu \mathrm{M}$ taxol and $1.3 \mathrm{mg} / \mathrm{mL}$ casein. Polarity-labeled TMR (tetramethylrhodamine) microtubules diluted in BRB50 supplemented with $20 \mu \mathrm{M}$ taxol and $1.3 \mathrm{mg} / \mathrm{mL}$ casein were then added to the chamber and unbound microtubules were removed with two chamber volumes of this buffer after a 2-min incubation. The flow chamber was then perfused with one chamber volume of BRB50 motility buffer containing $1 \mathrm{mM}$ ATP, $25 \mu \mathrm{M}$ taxol, $1.3 \mathrm{mg} / \mathrm{mL}$ casein, and an oxygen scavenger system. Time-lapse images were taken at one frame per $5 \mathrm{sec}$ for $5 \mathrm{~min}$. The measurements of 100 microtubules were collected from 14 different gliding experiments. Although the KINDR gliding assays published in Dawe et al. (2018) and the TRKIN assays reported here were not performed in parallel, they were carried out under similar conditions with the same microscopy system.

\section{Acknowledgments}

This study was supported by National Science Foundation grants 1412063 and 1925546 to R.K.D. and 1616462 to W.Q., and an NSF Doctoral Dissertation Improvement Grant to E.G.L. We relied on computational resources and technical expertise from the Georgia Advanced Computing Resource Center, a partnership between the University of Georgia's Office of the Vice President for Research and Office of the Vice President for Information Technology.

Author contributions: R.K.D. oversaw the project. K.W.S., J.I.G., and R.K.D. wrote the paper. K.W.S., V.S., and W.Q. produced the figures. J.I.G., E.G.L., and A.E.H. contributed to the discovery of Trkin. V.S. performed structured illumination microscopy. K.-F.T, X.R., and W.Q. carried out gliding assays. J.I.G. analyzed tandem repeat abundances. All other work was performed by K.W.S.

\section{References}

Akera T, Chmátal L, Trimm E, Yang K, Aonbangkhen C, Chenoweth DM, Janke C, Schultz RM, Lampson MA. 2017. Spindle asymmetry drives non-Mendelian chromosome segregation. Science 358: 668-672. doi:10.1126/science.aan0092

Akera T, Trimm E, Lampson MA. 2019. Molecular strategies of meiotic cheating by selfish centromeres. Cell 178: 11321144.e10. doi:10.1016/j.cell.2019.07.001

Albert PS, Gao Z, Danilova TV, Birchler JA. 2010. Diversity of chromosomal karyotypes in maize and its relatives. Cytogenet Genome Res 129: 6-16. doi:10.1159/000314342

Ananiev EV, Phillips RL, Rines HW. 1998. A knob-associated tandem repeat in maize capable of forming fold-back DNA segments: are chromosome knobs megatransposons? Proc Nat1 Acad Sci 95: 10785-10790. doi:10.1073/pnas.95.18.10785

Asai DJ, Thompson WC, Wilson L, Brokaw CJ. 1982. Two different monoclonal antibodies to $\alpha$-tubulin inhibit the bending of reactivated sea urchin spermatozoa. Cell Motil 2: 599-614. doi:10.1002/cm.970020608

Bell KM, Cha HK, Sindelar CV, Cochran JC. 2017. The yeast kinesin-5 Cin8 interacts with the microtubule in a noncanonical manner. I Biol Chem 292: 14680-14694. doi:10.1074/jbc .M117.797662

Buckler ES 4th, Phelps-Durr TL, Buckler CS, Dawe RK, Doebley JF, Holtsford TP. 1999. Meiotic drive of chromosomal knobs reshaped the maize genome. Genetics 153: 415-426.

Buonomo SB, Clyne RK, Fuchs J, Loidl J, Uhlmann F, Nasmyth K. 2000. Disjunction of homologous chromosomes in meiosis I depends on proteolytic cleavage of the meiotic cohesin Rec8 by separin. Cell 103: 387-398. doi:10.1016/S0092-8674(00) 00131-8

Charlesworth B, Sniegowski P, Stephan W. 1994. The evolutionary dynamics of repetitive DNA in eukaryotes. Nature 371: 215-220. doi:10.1038/371215a0

Chmátal L, Gabriel SI, Mitsainas GP, Martínez-Vargas J, Ventura J, Searle JB, Schultz RM, Lampson MA. 2014. Centromere strength provides the cell biological basis for meiotic drive and karyotype evolution in mice. Curr Biol 24: 2295-2300. doi:10.1016/j.cub.2014.08.017

Chmátal L, Yang K, Schultz RM, Lampson MA. 2015. Spatial regulation of kinetochore microtubule attachments by destabilization at spindle poles in meiosis I. Curr Biol 25: 1835-1841. doi:10.1016/j.cub.2015.05.013

Clark FJ. 1940. Cytogenetic studies of divergent meiotic spindle formation in Zea mays. Am J Bot 27: 547-559. doi:10.1002/j .1537-2197.1940.tb14716.x

Cross RA, McAinsh A. 2014. Prime movers: the mechanochemistry of mitotic kinesins. Nat Rev Mol Cell Biol 15: 257-271. doi: $10.1038 / \mathrm{nrm} 3768$

Dawe RK. 1998. Meiotic chromosome organization and segregation in plants. Annu Rev Plant Physiol Plant Mol Biol 49: 371-395. doi:10.1146/annurev.arplant.49.1.371

Dawe RK, Cande WZ. 1996. Induction of centromeric activity in maize by suppressor of meiotic drive 1. Proc Natl Acad Sci 93: 8512-8517. doi:10.1073/pnas.93.16.8512

Dawe RK, Hiatt EN. 2004. Plant neocentromeres: fast, focused, and driven. Chromosome Res 12: 655-669. doi:10.1023/B: CHRO.0000036607.74671.db

Dawe RK, Lowry EG, Gent JI, Stitzer MC, Swentowsky KW, Higgins DM, Ross-Ibarra J, Wallace JG, Kanizay LB, Alabady M, et al. 2018. A kinesin-14 motor activates neocentromeres to promote meiotic drive in maize. Cell 173: 839-850.e18. doi:10.1016/j.cell.2018.03.009

Dobin A, Davis CA, Schlesinger F, Drenkow J, Zaleski C, Jha S, Batut P, Chaisson M, Gingeras TR. 2013. STAR: ultrafast universal RNA-seq aligner. Bioinformatics 29: 15-21. doi:10 .1093/bioinformatics/bts635 
Endow SA, Kull FJ, Liu H. 2010. Kinesins at a glance. J Cell Sci 123: 3420-3424. doi:10.1242/jcs.064113

Evans MMS, Grossniklaus U. 2009. The maize megagametophyte. In: Handbook of maize: its biology (ed. Bennetzen JL, Hake SC), pp. 79-104. Springer New York, New York.

Fishman L, McIntosh M. 2019. Standard deviations: the biological bases of transmission ratio distortion. Annu Rev Genet 53: 347-372. doi:10.1146/annurev-genet-112618-043905

Fishman L, Saunders A. 2008. Centromere-associated female meiotic drive entails male fitness costs in monkeyflowers. Science 322: 1559-1562. doi:10.1126/science.1161406

Garrido-Ramos MA. 2017. Satellite DNA: an evolving topic. Genes 8: 230. doi:10.3390/genes8090230.

Gent JI, Wang N, Dawe RK. 2017. Stable centromere positioning in diverse sequence contexts of complex and satellite centromeres of maize and wild relatives. Genome Biol 18: 121. doi:10.1186/s13059-017-1249-4

Gerson-Gurwitz A, Thiede C, Movshovich N, Fridman V, Podolskaya M, Danieli T, Lakämper S, Klopfenstein DR, Schmidt CF, Gheber L. 2011. Directionality of individual kinesin-5 Cin 8 motors is modulated by loop 8 , ionic strength and microtubule geometry. EMBO J 30: 4942-4954. doi:10.1038/emboj .2011 .403

Ghaffari R, Cannon EKS, Kanizay LB, Lawrence CJ, Dawe RK. 2013. Maize chromosomal knobs are located in gene-dense areas and suppress local recombination. Chromosoma 122: 6775. doi:10.1007/s00412-012-0391-8

Gicking AM, Swentowsky KW, Dawe RK, Qiu W. 2018. Functional diversification of the kinesin-14 family in land plants. FEBS Lett 592: 1918-1928. doi:10.1002/1873-3468.13094

Gulick AM, Song H, Endow SA, Rayment I. 1998. X-ray crystal structure of the yeast Kar3 motor domain complexed with Mg.ADP to 2.3 A resolution. Biochemistry 37: 1769-1776. doi:10.1021/bi972504o

Hall DW, Dawe RK. 2018. Modeling the evolution of female meiotic drive in maize. G3 8: 123-130. doi:10.1534/g3.117.300073

Hiatt EN, Kentner EK, Dawe RK. 2002. Independently regulated neocentromere activity of two classes of tandem repeat arrays. Plant Cell 14: 407-420. doi:10.1105/tpc.010373

Higgins DM, Nannas NJ, Dawe RK. 2016. The maize divergent spindle-1 (dv1) gene encodes a kinesin-14A motor protein required for meiotic spindle pole organization. Front Plant Sci 7: 1277. doi:10.3389/fpls.2016.01277

Higgins DM, Lowry EG, Kanizay LB, Becraft PW, Hall DW, Dawe RK. 2018. Fitness costs and variation in transmission distortion associated with the abnormal chromosome 10 meiotic drive system in maize. Genetics 208: 297-305. doi:10.1534/ge netics.117.300060

Huang Y, Wang H, Huang X, Wang Q, Wang J, An D, Li J, Wang W, Wu Y. 2019. Maize VKS1 regulates mitosis and cytokinesis during early endosperm development. Plant Cell 31: 12381256. doi:10.1105/tpc. 18.00966

Hyman AA. 1991. Preparation of marked microtubules for the assay of the polarity of microtubule-based motors by fluorescence. J Cell Sci Suppl 1991: 125-127. doi:10.1242/jcs.1991 .Supplement_14.25

Iwata-Otsubo A, Dawicki-McKenna JM, Akera T, Falk SJ, Chmátal L, Yang K, Sullivan BA, Schultz RM, Lampson MA, Black BE. 2017. Expanded satellite repeats amplify a discrete CENP-A nucleosome assembly site on chromosomes that drive in female meiosis. Curr Biol 27: 2365-2373.e8. doi:10 .1016/j.cub.2017.06.069

Kanizay LB, Albert PS, Birchler JA, Dawe RK. 2013a. Intragenomic conflict between the two major knob repeats of maize. Genetics 194: 81-89. doi:10.1534/genetics.112.148882
Kanizay LB, Pyhäjärvi T, Lowry EG, Hufford MB, Peterson DG, Ross-Ibarra J, Dawe RK. 2013b. Diversity and abundance of the abnormal chromosome 10 meiotic drive complex in Zea mays. Heredity (Edinb) 110: 570-577. doi:10.1038/hdy.2013.2

Kato YTA. 1976. Cytological studies of maize (Zea mays L.) and teosinte (Zea mexicana Schrader Kuntze) in relation to their origin and evolution. Mass Agric Exp Stn Bull 635: 1-185.

Kozielski F, Sack S, Marx A, Thormählen M, Schönbrunn E, Biou V, Thompson A, Mandelkow EM, Mandelkow E. 1997. The crystal structure of dimeric kinesin and implications for microtubule-dependent motility. Cell 91: 985-994. doi:10 .1016/S0092-8674(00)80489-4

Lawrence CJ, Dawe RK, Christie KR, Cleveland DW, Dawson SC, Endow SA, Goldstein LSB, Goodson HV, Hirokawa N, Howard J, et al. 2004. A standardized kinesin nomenclature. J Cell Biol 167: 19-22. doi:10.1083/jcb.200408113

Li H. 2013. Aligning sequence reads, clone sequences and assembly contigs with BWA-MEM. arXiv [q-bioGN] http://arxiv .org/abs/1303.3997.

Li H. 2018. Minimap2: pairwise alignment for nucleotide sequences. Bioinformatics 34: 3094-3100. doi:10.1093/bioinfor matics/bty191

Li H, Handsaker B, Wysoker A, Fennell T, Ruan J, Homer N, Marth G, Abecasis G, Durbin R, 1000 Genome Project Data Processing Subgroup. 2009. The sequence alignment/map format and SAMtools. Bioinformatics 25: 2078-2079. doi:10 .1093/bioinformatics/btp352

Liu J, Seetharam AS, Chougule K, Ou S, Swentowsky KW, Gent JI, Llaca V, Woodhouse MR, Manchanda N, Presting GG, et al. 2020. Gapless assembly of maize chromosomes using longread technologies. Genome Biol 21: 121. doi:10.1186/ s13059-020-02029-9

Longley AE. 1938. Chromosomes of maize from North American Indians. J Agric Res 56: 177-195.

Longley AE. 1945. Abnormal segregation during megasporogenesis in maize. Genetics 30: 100-113.

Manoli A, Sturaro A, Trevisan S, Quaggiotti S, Nonis A. 2012. Evaluation of candidate reference genes for qPCR in maize. $J$ Plant Physiol 169: 807-815. doi:10.1016/j.jplph.2012.01.019

Marx A, Hoenger A, Mandelkow E. 2009. Structures of kinesin motor proteins. Cell Motil Cytoskeleton 66: 958-966. doi:10 $.1002 / \mathrm{cm} .20392$

McClintock B. 1929. Chromosome morphology in Zea mays. Science 69: 629. doi:10.1126/science.69.1798.629

McClintock B. 1939. The behavior in successive nuclear divisions of a chromosome broken at meiosis. Proc Natl Acad Sci 25: 405-416. doi:10.1073/pnas.25.8.405

McClintock B. 1941. The stability of broken ends of chromosomes in Zea mays. Genetics 26: 234-282.

Minh BQ, Schmidt HA, Chernomor O, Schrempf D, Woodhams MD, von Haeseler A, Lanfear R. 2020. IQ-TREE 2: new models and efficient methods for phylogenetic inference in the genomic era. Mol Biol Evol 37: 1530-1534. http://dx.doi.org/10 $.1093 / \mathrm{molbev} / \mathrm{msaa} 015$.

Mroczek RJ, Melo JR, Luce AC, Hiatt EN, Dawe RK. 2006. The maize Ab10 meiotic drive system maps to supernumerary sequences in a large complex haplotype. Genetics 174: 145-154. doi:10.1534/genetics.105.048322

Novák P, Neumann P, Macas J. 2010. Graph-based clustering and characterization of repetitive sequences in next-generation sequencing data. BMC Bioinformatics 11: 378. doi:10.1186/ 1471-2105-11-378

Novitski E. 1952. The genetic consequences of anaphase bridge formation in Drosophila. Genetics 37: 270-287. 
Peacock WJ, Dennis ES, Rhoades MM, Pryor AJ. 1981. Highly repeated DNA sequence limited to knob heterochromatin in maize. Proc Natl Acad Sci 78: 4490-4494. doi:10.1073/pnas 78.7.4490

Popchock AR, Tseng K-F, Wang P, Karplus PA, Xiang X, Qiu W. 2017. The mitotic kinesin-14 KlpA contains a context-dependent directionality switch. Nat Commun 8: 13999. doi:10 $.1038 /$ ncomms 13999

Rhoades MM. 1942. Preferential segregation in maize. Genetics 27: 395-407.

Rhoades MM. 1952. Preferential segregation in maize. In Heterosis (ed. Gowen JW), pp. 66-80. Iowa State College Press, Ames, IA.

Robinson JT, Thorvaldsdóttir H, Winckler W, Guttman M, Lander ES, Getz G, Mesirov JP. 2011. Integrative genomics viewer. Nat Biotechnol 29: 24-26. doi:10.1038/nbt.1754

Russell SD. 1979. Fine structure of megagametophyte development in Zea mays. Can I Bot 57: 1093-1110. doi:10.1139/ b79-134

Vera Alvarez R, Pongor LS, Mariño-Ramírez L, Landsman D. 2019. TPMCalculator: one-step software to quantify mRNA abundance of genomic features. Bioinformatics 35: 19601962. doi:10.1093/bioinformatics/bty896
Weisshart K, Fuchs J, Schubert V. 2016. Structured illumination microscopy (SIM) and photoactivated localization microscopy (PALM) to analyze the abundance and distribution of RNA polymerase II molecules on flow-sorted Arabidopsis nuclei. Bio Protoc 6: e1725. doi:10.21769/BioProtoc. 1725

Woehlke G, Ruby AK, Hart CL, Ly B, Hom-Booher N, Vale RD. 1997. Microtubule interaction site of the kinesin motor. Cell 90: 207-216. doi:10.1016/S0092-8674(00)80329-3

Wu T, Lane SIR, Morgan SL, Jones KT. 2018. Spindle tubulin and MTOC asymmetries may explain meiotic drive in oocytes. Nat Commun 9: 2952. doi:10.1038/s41467-018-05338-7

Yamagishi M, Shigematsu H, Yokoyama T, Kikkawa M, Sugawa M, Aoki M, Shirouzu M, Yajima J, Nitta R. 2016. Structural basis of backwards motion in kinesin-1-kinesin-14 chimera: implication for kinesin-14 motility. Structure 24: 13221334. doi:10.1016/j.str.2016.05.021

Yu HG, Hiatt EN, Chan A, Sweeney M, Dawe RK. 1997. Neocentromere-mediated chromosome movement in maize. I Cell Biol 139: 831-840. doi:10.1083/jcb.139.4.831

Zhang H, Dawe RK. 2011. Mechanisms of plant spindle formation. Chromosome Res 19: 335-344. doi:10.1007/s10577011-9190-y 


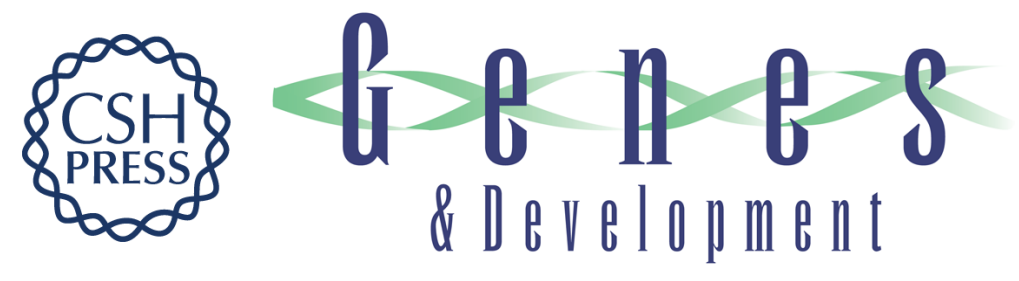

\title{
Distinct kinesin motors drive two types of maize neocentromeres
}

Kyle W. Swentowsky, Jonathan I. Gent, Elizabeth G. Lowry, et al.

Genes Dev. 2020, 34: originally published online August 20, 2020

Access the most recent version at doi:10.1101/gad.340679.120

\author{
Supplemental http://genesdev.cshlp.org/content/suppl/2020/08/20/gad.340679.120.DC1 \\ Material \\ Related Content Mixed knobs in corn cobs \\ Piero Lamelza and Michael A. Lampson \\ Genes Dev. September , 2020 34: 1110-1112
}

References This article cites 65 articles, 22 of which can be accessed free at: http://genesdev.cshlp.org/content/34/17-18/1239.full.html\#ref-list-1

Articles cited in: http://genesdev.cshlp.org/content/34/17-18/1239.full.html\#related-urls

Creative This article is distributed exclusively by Cold Spring Harbor Laboratory Press for the first Commons six months after the full-issue publication date (see

License http://genesdev.cshlp.org/site/misc/terms.xhtml). After six months, it is available under a Creative Commons License (Attribution-NonCommercial 4.0 International), as described at http://creativecommons.org/licenses/by-nc/4.0/.

Email Alerting Receive free email alerts when new articles cite this article - sign up in the box at the top Service right corner of the article or click here.

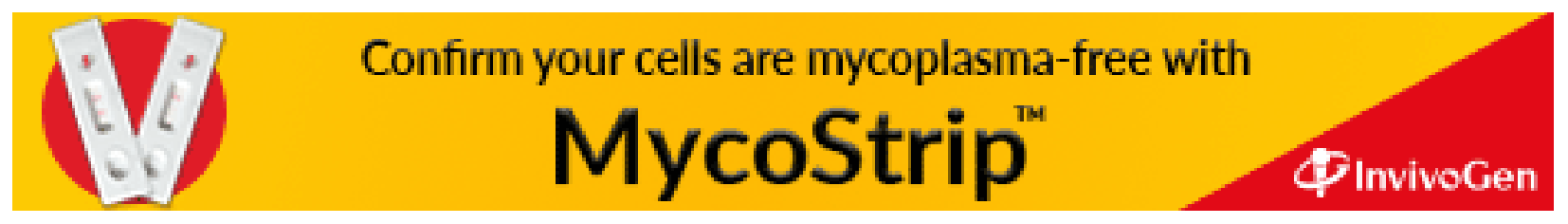

